\title{
Estudo dos Parâmetros de União por Difusão do Aço Inoxidável AISI 316L
}

\author{
Marcus Vinícius Volponi Mortean ${ }^{1 *}$ (D), Luisa Bastos Mateus², Grégori Rosinski², Marcia Barbosa Henriques Mantelli² \\ ${ }^{1}$ Universidade Federal de Santa Catarina - UFSC, Departamento de Engenharia da Mobilidade, Joinville, SC, Brasil. \\ 2 Universidade Federal de Santa Catarina - UFSC, Departamento de Engenharia Mecânica, Laboratório de Tubos de Calor, Florianopolis, SC, Brasil.
}

Como citar: Mortean MVV, Mateus LB, Rosinski G, Mantelli MBH. Estudo dos parâmetros de união por difusão do aço inoxidável AISI 316L. Soldagem \& Inspeção. 2019;24:e2409. https://doi.org/10.1590/0104-9224/SI24.09

\begin{abstract}
Resumo: A união por difusão é uma técnica de união no estado sólido capaz de ligar dois materiais metálicos por meio da difusão atômica na interface de contato, sem o uso de metal de adição. Este processo é adequado para a união de grandes áreas com uma alta resistência mecânica, permitindo ao equipamento operar com elevadas pressões de trabalho, características essenciais em aplicações com mini e micro canais, como em trocadores de calor compactos e reatores. O presente trabalho tem como objetivo estudar a influência dos principais parâmetros de união: temperatura, pressão e tempo, na união de uma junta de aço inoxidável AISI 316L/316L, além de encontrar o conjunto de parâmetros que apresente os melhores resultados. No total, 9 conjuntos de parâmetros foram estudados. As amostras foram avaliadas por ensaios de tração e análises micro estruturais da interface de união. Uma amostra, do metal base na condição de fornecimento ("as received"), foi usada como padrão para as comparações. Com base nos resultados, verificou-se que o parâmetro temperatura foi o que apresentou a maior influência na qualidade da união e que as amostras produzidas com $1040{ }^{\circ} \mathrm{C}$ apresentaram resistência mecânica da mesma ordem do metal base.
\end{abstract}

Palavras-chave: União por difusão; Aço inoxidável; Parâmetros ótimos de união; Trocador de calor compacto.

\section{Study of Diffusion Bonding Parameters of AISI 316L Stainless Steel}

Abstract: Diffusion bonding process is a solid state joining technique capable of joining two flat metal surfaces by diffusion of atoms in the contact interface, without the use of addition metal. This process can bond large areas with a high mechanical strength at the bonded interfaces, allowing the equipment to operate at high working pressures, which is an essential characteristic in applications involving mini and micro channels, such as compact heat exchangers and reactors. The present work focus on the study of the influence of the main welding parameters: temperature, pressure and time, in the join of stainless steel AISI 316L/316L plates, in addition to finding the set of parameters that present the best union results. In total, 9 sets of parameters were studied. The samples were evaluated by tensile tests and microstructural analysis of the bonding interface. A sample of the base metal was used as the standard for comparison. Based on the tensile test results, it was verified that the temperature parameter presented the highest influence on the bond quality, and that the samples produced at $1040{ }^{\circ} \mathrm{C}$ temperature level showed mechanical strength of the same order of the base material.

Key-words: Diffusion bonding; Stainless steel; Optimal join parameters; Compact heat exchanger.

\section{Introdução}

Trocadores de calor compactos, micro reatores e micro células de combustível são sistemas que podem ser fabricados pelo empilhamento alternado de placas finas com micro/mini canais. O processo de união do conjunto precisa ser preciso, de modo a não bloquear os canais de passagem do escoamento. Portanto as técnicas convencionais de soldagem por fusão dificilmente são capazes de satisfazer as necessidades de união requisitadas nessas aplicações [1,2].

União por difusão mostra ser uma boa alternativa [3] quando são exigidos altos requisitos dimensionais e de qualidade na união de microssistemas. A efetividade e as pequenas distorções resultantes da aplicação deste processo têm sido demonstradas com sucesso em diversos casos, tanto para metais similares quanto para dissimilares [1].

Essa técnica permite a união de grandes áreas, além de conferir ao conjunto grande resistência mecânica na região unida, permitindo ao equipamento operar com elevadas pressões de trabalho [3-5]. Trocadores de calor de circuito impresso [6,7], placas aletadas [8] e placas cortadas [9] são exemplos de trocadores de calor compactos fabricados a partir de processos de união por difusão, os quais foram desenvolvidos principalmente para aplicações onde o espaço é restrito, as pressões de trabalho são altas e os fluidos empregados são corrosivos [4]. 
A união por difusão é um processo que ocorre no estado sólido, obtido através de ligações em nível atômico de duas superfícies preparadas. Essa união está relacionada com deformações plásticas locais devido à aplicação de altas temperaturas e pressões externas, que auxiliam no aumento do contato superficial e na difusão atômica, tendo como resultado o fechamento das superfícies de contato. Visando evitar deformações macroscópicas, as pressões de união são normalmente consideravelmente inferiores à tensão de escoamento na temperatura ambiente. A temperatura necessária para a união normalmente está entre 0,5 e 0,8 da temperatura de fusão do material e o tempo pode variar de alguns minutos a diversas horas $[8,10]$.

Segundo An e Tu [11] aços inoxidáveis são uma boa alternativa para aplicações em microssistemas, uma vez que apresentam excelentes propriedades mecânicas e boa resistência à corrosão. Nos últimos anos houve um grande interesse na aplicação dos aços inoxidáveis austeníticos para a fabricação de reatores e vasos de pressão em aplicações nas indústrias química e nuclear $[12,13]$.

Diversos estudos da união por difusão de aços inoxidáveis com materiais dissimilares foram realizados visando principalmente as aplicações anteriormente mencionadas. Entretanto, existe um número limitado de trabalhos na literatura a respeito da união por difusão entre peças de aços inoxidáveis [11]. Dentre os trabalhos existentes, podemos citar o de Mortean et al. [14]. Os autores realizam um estudo experimental da soldagem por difusão de diferentes tipos de aços inoxidáveis: austenítico AISI 316L, duplex UNS 31803 e superduplex UNS 327250, focado na fabricação de trocadores de calor compactos. Como trocadores de calor compactos soldados por difusão operam em altas pressões de trabalho, a qualidade da interface de união é extremamente importante, falhas não identificadas podem comprometer a resistência mecânica e resultar em uma redução da pressão máxima de operação. Entretanto, os trabalhos existentes na literatura divergem sobre as melhores condições de união por difusão de aços inoxidáveis, como se mostra a seguir.

Pavlova [15] estudou a união do aço inoxidável 304 e adotou inicialmente, três valores de temperaturas $\left(850{ }^{\circ} \mathrm{C}, 1000{ }^{\circ} \mathrm{C}\right.$ e $1100^{\circ} \mathrm{C}$ ) para uma faixa de pressão de $12 \mathrm{MPa}$ até $17 \mathrm{MPa}$, e tempo de $30 \mathrm{~min}$. Para $850^{\circ} \mathrm{C}$, a autora não conseguiu a junção completa das amostras. Para $1100^{\circ} \mathrm{C}$, ocorreu excessiva deformação plástica das amostras e endurecimento do material base. Em seguida, para uma temperatura de $1000^{\circ} \mathrm{C}$ e pressão de $17 \mathrm{MPa}$, empregou três intervalos de tempos distintos: 30,40 e 60 min. Segundo a autora, o tempo de união de 30 min não foi suficiente para completar o estágio final da união por difusão, resultando em uma fratura frágil durante os testes de tração. Já um tempo de 40 min foi suficiente para completar a união. Além disso, observou que o aumento do tempo de união de 40 para 60 min não influenciou significativamente as propriedades da junção.

An et al. [1] realizaram um estudo aprofundado a respeito da união por difusão do aço inoxidável AISI 316L. Os parâmetros de união foram similares aos utilizados por Pavlova [15]. Utilizaram uma pressão constante de 10 MPa durante 60 minutos, variando a temperatura em 3 níveis: 850, 950 e $1050^{\circ} \mathrm{C}$. Os testes de tração foram realizados em dois níveis de temperaturas: ambiente e $550^{\circ} \mathrm{C}$. De acordo com os autores, a maior resistência mecânica foi obtida com uma temperatura de união de $850{ }^{\circ} \mathrm{C}$. Entretanto, houve uma queda significativa na resistência da junção quando ensaiada à elevada temperatura, correspondendo a apenas $37 \%$ da resistência da mesma união ensaiada à temperatura ambiente.

An e Tu [11] analisaram os modos de falha de amostras de aço inoxidável 316L unidas por difusão, com uma pressão constante de $10 \mathrm{Mpa}$. Os parâmetros tempo e temperatura foram analisados. Na primeira parte do trabalho foi estudada a influência da temperatura na resistência mecânica da união, para quatro níveis de temperatura: $850{ }^{\circ} \mathrm{C}, 950{ }^{\circ} \mathrm{C}, 1050{ }^{\circ} \mathrm{C}$ e $1100{ }^{\circ} \mathrm{C}$, para um tempo de $60 \mathrm{~min}$. Na segunda etapa foi estudada a influência do tempo na união dos metais: outras duas amostras foram unidas a $1100{ }^{\circ} \mathrm{C}$, com um tempo de $2 \mathrm{~h}$ e $3 \mathrm{~h}$. Os resultados mostraram que a razão de ligação (área de superfície unida) aumenta à medida que a temperatura aumenta. Além disso, os autores constataram que um tempo de união longo pode resultar em um excelente alongamento da amostra e uma alta razão de união. Por outro lado, também pode resultar em uma degradação da resistência da junta, atribuída à formação de FeCr.

Li et al. [3] empregaram os parâmetros utilizados por An e Tu [11] na união por difusão do aço inoxidável 316L. A união foi realizada com temperatura de $1100^{\circ} \mathrm{C}, 10 \mathrm{MPa}$ e $180 \mathrm{~min}$. Os resultados mostraram que a resistência a tração da amostra soldada por difusão foi próxima da resistência do metal base, com uma diferença de aproximadamente $6 \%$ entre as duas amostras.

Como pode ser visto, existem diferenças entre os parâmetros de união utilizados nos estudos encontrados na literatura. Em razão dessa diferença e visando garantir uma união integra e sem falhas, no presente trabalho é realizado um estudo experimental dos três parâmetros de união: temperatura, pressão e tempo, do aço inoxidável 316L. O objetivo é verificar a influência dos principais parâmetros: temperatura, pressão e tempo, na união de uma junta de aço inoxidável AISI 316L/316L, além de encontrar o conjunto de parâmetros que resulta em uma solda integra. Diversas amostras foram produzidas variando estes três parâmetros, as quais foram avaliadas por ensaios de tração, baseados na norma ASTM E8 [16], e por análises micro estruturais da interface de união.

\section{Materiais e Métodos}

Para atingir os objetivos da presente pesquisa, 10 amostras foram produzidas, usando o forno prensa de alto vácuo PVA Tepla MOV 653 HP, disponível no Laboratório de Tubos de Calor/UFSC, como pode ser visto na (Figura 1a). O forno possui um 
volume útil de $800 \times 600 \times 600 \mathrm{~mm}^{3}$, com uma capacidade de carga máxima de 250 ton e uma temperatura de operação máxima de $1700^{\circ} \mathrm{C}$.

Como já mencionado, os três principais parâmetros de processo, que afetam a qualidade da junta soldada por difusão, são: temperatura, pressão e tempo. Com base em artigos já mencionados $[1,3,11,15]$ e nos resultados obtidos por Mortean et al. [14], os níveis de operação de cada parâmetro foram identificados e empregados nas soldagens da presente pesquisa.

A máxima pressão de união empregada no presente trabalho é de $10 \mathrm{MPa}$ (limitação do forno), sendo o valor mínimo de 7,5 MPa e um nível intermediário de $8,75 \mathrm{MPa}$. Como a temperatura de união não deve ultrapassar 0,8 da temperatura de fusão do material, temperaturas entre $945^{\circ} \mathrm{C}$ e $1100^{\circ} \mathrm{C}$, com um nível intermediário de $1040{ }^{\circ} \mathrm{C}$, foram utilizadas. Com base nos valores disponíveis na literatura, estipulou-se que o tempo de união variaria entre 32 e $180 \mathrm{~min}$.
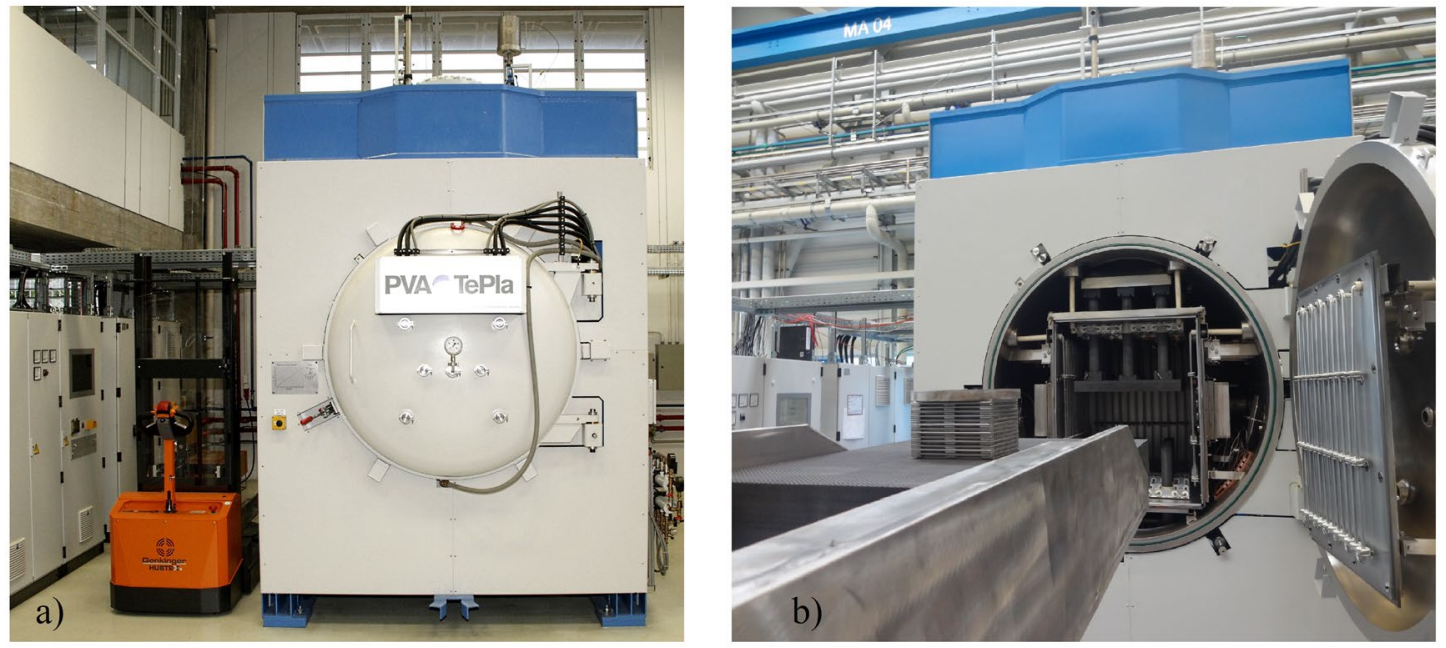

Figura 1. a) Forno prensa de união por difusão, b) câmara de união.

As amostras foram produzidas a partir da união por difusão de dois blocos de aço inoxidável AISI 316L de 47,5 mm de altura, $110 \mathrm{~mm}$ de largura e $110 \mathrm{~mm}$ de comprimento. As interfaces de contato entre blocos foram polidas e os blocos, posicionadas um sobre o outro (Figura 2a), foram inseridos no forno prensa de alto vácuo (Figura 1b). Visando proteger as matrizes do forno e evitar a união entre elas e a amostra, foi aplicada uma fina camada de nitrato de boro nas superfícies externas dos blocos. Após a união, foram retiradas do bloco unido três amostras cilíndricas, por meio do corte a jato de água, para o ensaio de tração, como mostra a Figura $2 b$, e uma amostra retangular para metalografia.
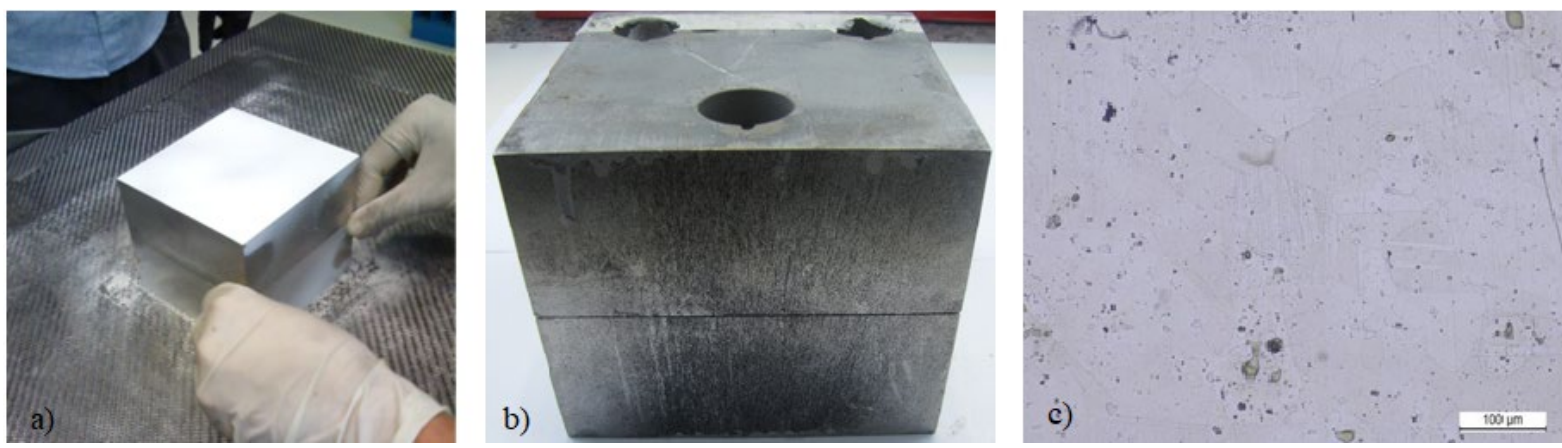

Figura 2. a) Blocos preparados para união, b) Corte das amostras e c) metalografia do metal base na condição de "as received".

A região unida da amostra retangular foi preparada para metalografia. As lixas de tamanho de grão 100, 220, 600, 1200 e 1500 foram usadas sequencialmente. Após o lixamento, as peças foram polidas com pasta diamantada de $1 \mu \mathrm{m}$.

Após o processo de polimento, os espécimes foram quimicamente atacados com gliceregia, que consiste numa mistura na composição: $10 \mathrm{~mL}$ de HNO3, $15 \mathrm{~mL}$ de ácido acético, $10 \mathrm{~mL}$ de $\mathrm{HCl}$ e 3 gotas de glicerina. Após o condicionamento, as microestruturas resultantes de cada ligação foram analisadas em microscópio óptico.

Os ensaios de tração foram realizados de acordo com a norma ASTM E8 [16] para amostras cilíndricas de pequeno porte, resultantes do corte do bloco soldado. As dimensões das amostras estão apresentadas na Figura 3a, sendo que a interface de união está localizada na região central da amostra. A Figura 3b apresenta o corpo de prova S1 após o ensaio de tração. 


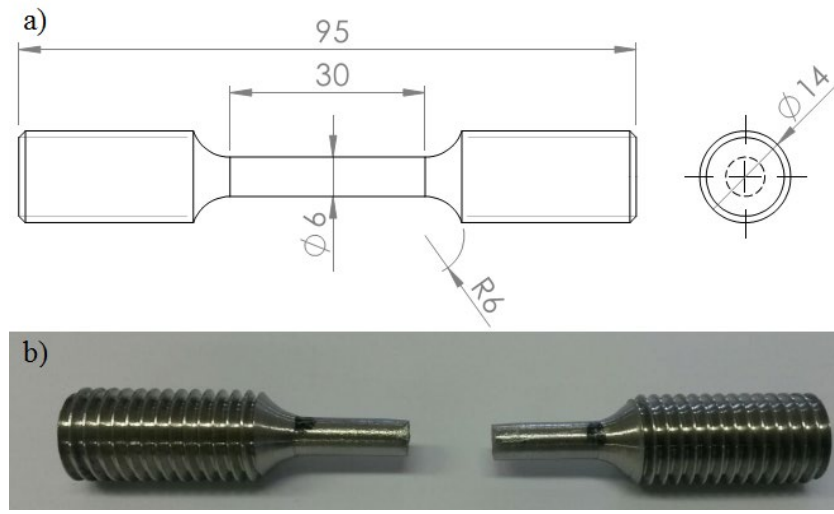

Figura 3. a) Dimensões da amostra do ensaio de tração, b) Amostra S1 após o ensaio.

No total foram realizadas nove uniões, sendo extraídos três corpos de prova de cada bloco resultante do processo de união. A denominação dos corpos de prova testados e os parâmetros de união são apresentados na Tabela 1 . A fim de avaliar o nível da união e compará-lo com um resultado padrão, corpos de prova sem tratamento térmico, provenientes do metal base na condição em que foi fornecido pela empresa, denominado "as received", foram produzidos e submetidos a ensaios de tração. No total 29 corpos de prova foram usinados. Note-se que somente dois corpos de prova foram retirados da amostra So, uma vez que o terceiro quebrou-se durante o processo de usinagem.

Tabela 1. Parâmetros de união por difusão utilizados.

\begin{tabular}{|c|c|c|c|}
\hline \multirow{2}{*}{ Amostra } & Temperatura & Pressão & Tempo \\
\hline & $\mathrm{T}\left({ }^{\circ} \mathrm{C}\right)$ & $\mathrm{P}$ (MPa) & $t(\min )$ \\
\hline So & & As received & \\
\hline S1 & 945 & 8,75 & 105 \\
\hline S2 & 945 & 7,5 & 105 \\
\hline S3 & 945 & 10 & 105 \\
\hline S4 & 945 & 8,75 & 32 \\
\hline S5 & 945 & 8,75 & 179 \\
\hline S6 & 1040 & 9,51 & 60 \\
\hline S7 & 1040 & 9,51 & 150 \\
\hline S8 & 1040 & 7,98 & 150 \\
\hline S9 & 1100 & 8,75 & 105 \\
\hline
\end{tabular}

O mesmo ciclo foi empregado no processo de união em todas as amostras produzidas. Uma atmosfera controlada com alto vácuo foi mantida durante o processo de união, com vácuo de pelo menos $5 \times 10^{-5} \mathrm{mbar}$. As amostras foram aquecidas até $600{ }^{\circ} \mathrm{C}$, à uma taxa de aquecimento de $5{ }^{\circ} \mathrm{C} / \mathrm{min}$ e mantidas nessa temperatura por $60 \mathrm{~min}$, permitindo a homogeneização da sua temperatura no interior da câmara. Em seguida, a amostra foi aquecida até a temperatura de união, com uma taxa de aquecimento de $2,5^{\circ} \mathrm{C} / \mathrm{min}$, e mantidas nessa temperatura durante todo tempo de união. Ao atingir a temperatura de união, aplicou-se a pressão externa, que foi mantida constante durante todo o tempo. O ciclo base de união é apresentado a seguir:

- Rampa 1: $20^{\circ} \mathrm{C}$ até $600{ }^{\circ} \mathrm{C}$, na taxa de $5^{\circ} \mathrm{C} / \mathrm{min}$

- Patamar 1: $600^{\circ} \mathrm{C}$ por $60 \mathrm{~min}$

- Rampa 2: $600^{\circ} \mathrm{C}$ até temperatura de união, na taxa de $2,5^{\circ} \mathrm{C} / \mathrm{min}$

- Patamar 2: Aplicação de diferentes parâmetros de temperatura, tempo e pressão externa.

Todo o processo de aquecimento foi controlado por meio de um termopar inserido na base da amostra, no interior do forno. Portanto, a temperatura empregada no presente trabalho refere-se exatamente à temperatura da amostra e não à temperatura interna do forno.

\section{Resultados e Discussões}

Os corpos de prova produzidos foram submetidos a ensaios de tração, que têm como objetivo identificar a carga máxima suportada pelo material até a sua ruptura. Os ensaios foram realizados em uma máquina universal de ensaios mecânicos EMIC (DL-10000), e os resultados obtidos são apresentados na Figura 4. A Figura 3b apresenta o corpo de prova 1 da amostra S1 após a sua ruptura. 
Para facilitar a comparação das amostras com o padrão (amostra SO - metal "as received" sem união), foram traçadas três linhas horizontais: uma (em preto) representando a média e outras duas (em cinza) mostrando o desvio padrão em relação à amostra padrão SO.

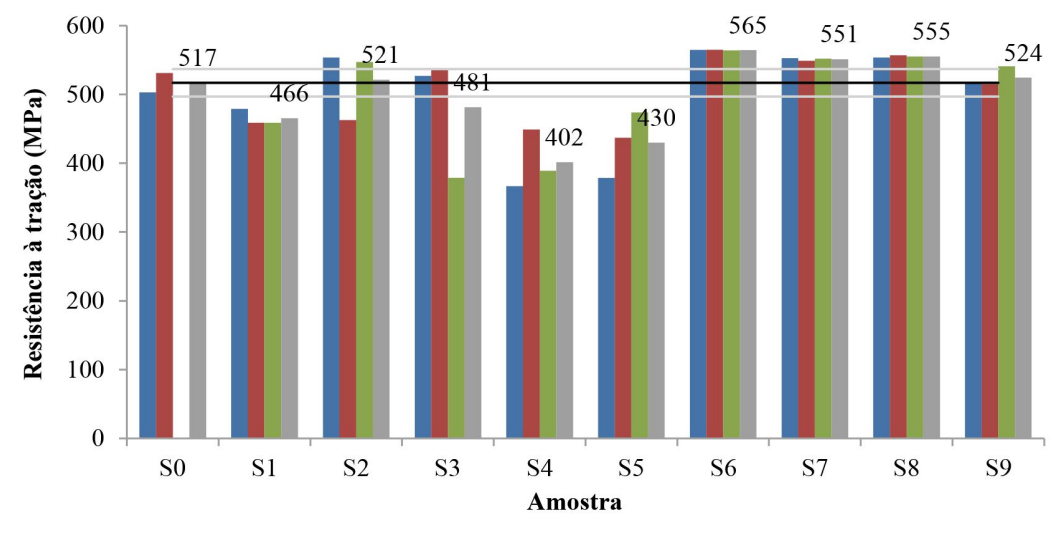

CP1 CP2 CP3 Média C Média metal "as received" —Desvio padrão

Figura 4. Resultado dos ensaios de tração.

\subsection{Influência dos parâmetros de união}

Para avaliar os parâmetros de união, cinco comparações entre as amostras foram realizadas. A Tabela 2 apresenta a comparação entre amostras, com o parâmetro de união analisado, ou seja, temperatura, pressão ou tempo. A combinação entre as amostras e o parâmetro a ser analisado define os "casos" (ver primeira coluna). Assim, nos Casos 1 e 2, avalia-se a influência da pressão, uma vez que os dois outros parâmetros (temperatura e tempo) foram mantidos constantes. Já nos Casos 3 e 4, investiga-se a influência do tempo de união e, no Caso 5, a influência da temperatura.

Tabela 2. Comparação entre as amostras.

\begin{tabular}{|c|c|c|c|c|c|}
\hline Parâmetro avaliado & Nome & Temp. $\left({ }^{\circ} \mathrm{C}\right)$ & Pressão (MPa) & Tempo (min) & $\begin{array}{l}\text { Resistência mecânica } \\
\text { média (MPa) }\end{array}$ \\
\hline \multirow[t]{3}{*}{ Caso 1) Pressão } & S2 & 945 & 7,5 & 105 & 521 \\
\hline & S1 & & 8,75 & & 466 \\
\hline & S3 & & 10 & & 481 \\
\hline \multirow[t]{2}{*}{ Caso 2) Pressão } & S8 & 1040 & 7,98 & 150 & 555 \\
\hline & S7 & & 9,51 & & 551 \\
\hline \multirow[t]{3}{*}{ Caso 3) Tempo } & S4 & 945 & 8,75 & 32 & 402 \\
\hline & S1 & & & 105 & 466 \\
\hline & S5 & & & 179 & 430 \\
\hline \multirow[t]{2}{*}{ Caso 4) Tempo } & S6 & 1040 & 9,51 & 60 & 565 \\
\hline & S7 & & & 150 & 551 \\
\hline \multirow[t]{2}{*}{ Caso 5) Temperatura } & S1 & 945 & 8,75 & 105 & 466 \\
\hline & S9 & 1100 & & & 524 \\
\hline
\end{tabular}

Os resultados dos Casos 1 e 2 mostram que a variação da pressão não influenciou significativamente a resistência mecânica, uma vez que os valores observados se mantiveram próximos nos casos analisados. No caso 1 , a variação da pressão resultou em uma diferença média da resistência mecânica, entre as três amostras, de 37 MPa para pressões variando de 7,5 a $10 \mathrm{MPa}$. Já no caso 2 a diferença entre elas foi de apenas $4 \mathrm{MPa}$, para uma variação de 7,98 a 9,51 MPa. Entretanto, essa pequena variação na resistência mecânica deve-se também ao fato de que a análise foi realizada em um intervalo bastante estreito de pressão, de 7,5 a 10MPa, que aparentemente não foi significativo para influenciar nos resultados.

Os resultados dos Casos 3 e 4 permitem verificar a influência do tempo de união na resistência mecânica. Novamente constatou-se que a diferença entre as amostras foi pequena. Para o Caso 3, a diferença média foi de $43 \mathrm{MPa}$, para tempos variando de 32 a 179 min e no Caso 4 a diferença máxima foi de $14 \mathrm{MPa}$, para tempos de 60 e $150 \mathrm{~min}$.

Entretanto, comparando o Caso 1 com 2 e o Caso 3 com o 4, verifica-se que o aumento da temperatura teve um resultado significativo na resistência. A resistência mecânica média no Caso 1 (amostras soldadas a $945^{\circ} \mathrm{C}$ ) foi de $489 \mathrm{MPa}$ e no Caso 2 (amostras soldadas a $1040{ }^{\circ} \mathrm{C}$ ) foi de $553 \mathrm{MPa}$, ou seja, o aumento da temperatura, de cerca de $100^{\circ} \mathrm{C}$, para níveis de pressão 
próximos, teve como resultado um aumento de $64 \mathrm{MPa}$ na resistência da amostra. 0 mesmo aumento foi identificado comparando os Casos 3 e 4, em que a resistência mecânica média do Caso 3 (temperatura de união de $945^{\circ} \mathrm{C}$ ) foi de $432 \mathrm{MPa}$ e a do Caso 4 (soldado com $1040^{\circ} \mathrm{C}$ ) foi de $558 \mathrm{MPa}$, ou seja, um aumento de $126 \mathrm{MPa}$, para um aumento de temperatura de $95^{\circ} \mathrm{C}$.

A influência da temperatura tornou-se evidente no Caso 5. Neste, os parâmetros tempo e pressão foram mantidos constantes, sendo estudado o efeito da temperatura na resistência mecânica da solda. Um aumento de temperatura, de $155^{\circ} \mathrm{C}$, resultou em um aumento da resistência mecânica de aproximadamente $60 \mathrm{MPa}$.

Dessa maneira, pôde-se constatar que a temperatura influenciou diretamente na qualidade da união em termos de resistência mecânica, assim como havia sido demonstrado nas pesquisas realizadas por An e Tu [11] e Pavlova [15]. Esta influência deve-se ao fato de que processos termicamente ativados, como difusão e fluência, variam exponencialmente com a temperatura [17].

Além disso, também foi medida a deformação dos corpos de prova, visto que o tamanho do alongamento é proporcional à ductilidade do material. Os resultados mostraram que as amostras S1 a S5 apresentaram um comportamento frágil, com deformações médias de 10\%, 25\%, 18\%, 5\% e 10\%, respectivamente, valores bem inferiores ao da amostra padrão S0 (37\%). A amostra S9 apresentou um comportamento similar ao do metal base (amostra S0), embora ainda tenha apresentado uma deformação média um pouco menor, de 30\%. As amostras S6, S7 e S8 tiveram um comportamento mais dúctil do que a amostra padrão, com deformações médias de $48 \%, 58 \%$ e $60 \%$, respectivamente. Além disso, foi possível constatar que o material não perdeu sua ductilidade, visto que a amostra S10 apresentou uma deformação média de 38\%.

\subsection{Análise microestrutural}

Visando uma análise mais completa da interface de união, uma análise microestrutural foi realizada, de modo a avaliar, de maneira qualitativa, a qualidade da união. Ao analisar os corpos de prova no microscópio óptico, foi possível observar a interface de união das placas, apresentadas nas imagens da Figura 5.

Na união de dois corpos de um mesmo material, a de qualidade da ligação está inversamente relacionada com a nitidez da linha de interface, ou seja, quanto mais nítida, menor é a qualidade da ligação.

Comparando as micrografias da Figura 5, é possível observar que, em alguns casos, a linha tornou-se mais evidente que em outros. Nota-se que as amostras S1 a S5 e S9 têm linhas de ligação mais nítidas quando comparadas com a amostra S6, S7 e S8. Isso significa que as amostras S6, S7 e S8 apresentaram maior continuidade de material na região ligada, em comparação com as outras.

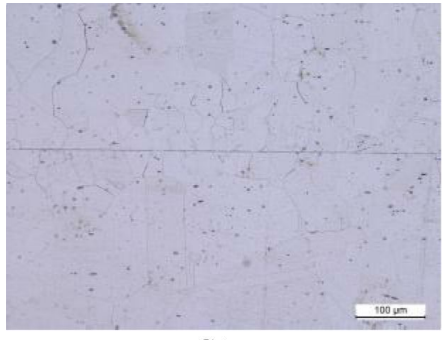

S1

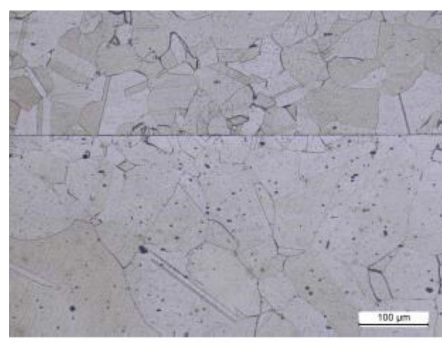

S4

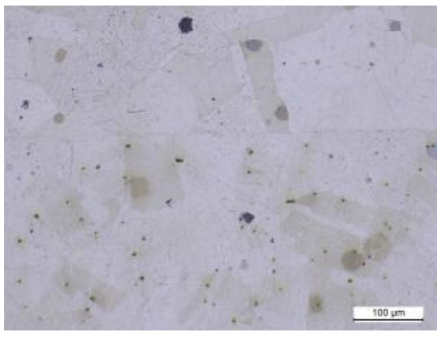

S7

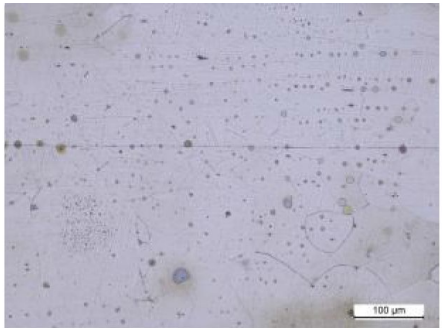

S2

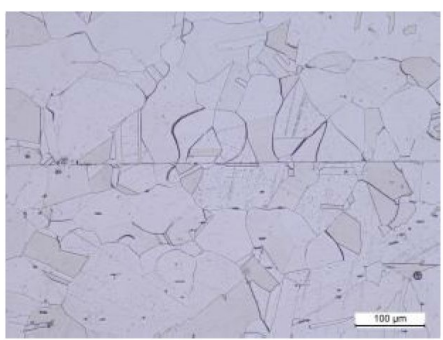

S5

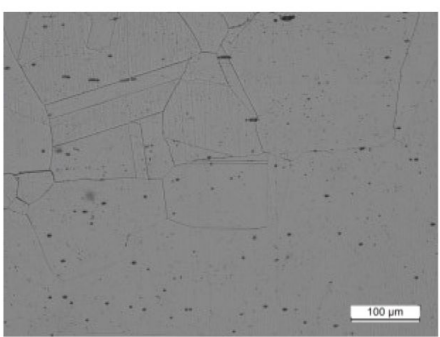

S8

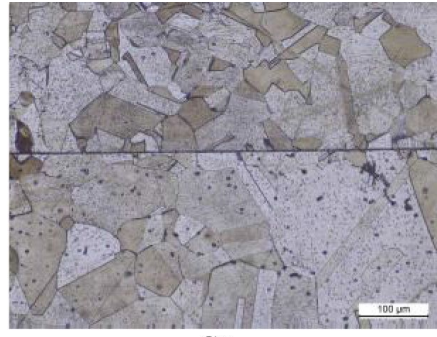

S3

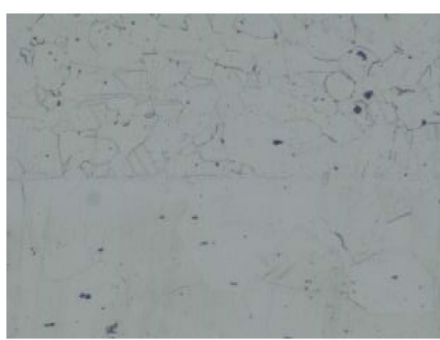

S6

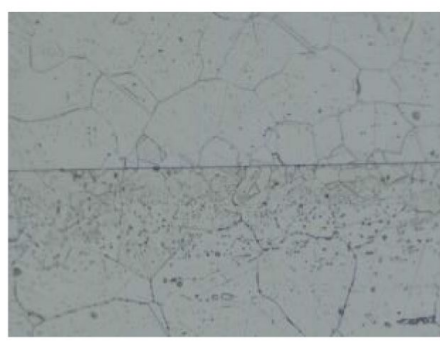

S9

Figura 5. Microscopia ótica das amostras soldadas. 


\subsection{Melhores parâmetros de união}

A Figura 4 apresenta o resultado dos ensaios mecânicos de tração para as nove amostras soldadas (S1 até S9), além da resistência mecânica do metal base (SO), sem nenhum tratamento ("as received").

Segundo a ASTM Standard A240 [18], o aço inoxidável 316L deve apresentar uma resistência a tração de pelo menos $515 \mathrm{MPa}$. O metal base submetido ao ensaio de tração apresentou uma resistência mecânica média de $517 \mathrm{MPa}$, maior que o mínimo estipulado pela norma.

Dentre as amostras fabricadas, apenas cinco apresentaram uma resistência mecânica maior que o mínimo estipulado pela norma: S2, S6, S7, S8 e S9. Isto comprova a alta qualidade da junta produzida com a técnica de união por difusão em termos de resistência mecânica e evidencia que a escolha dos parâmetros de união foi adequada, pois as propriedades mecânicas dos materiais unidos são similares às do próprio metal base.

Os melhores resultados foram obtidos com as três amostras soldadas com $1040{ }^{\circ} \mathrm{C}$ : S6, S7 e S8, visto que, além de apresentarem uma resistência média elevada, 565, 551 e $555 \mathrm{MPa}$, respectivamente, também apresentaram uma pequena variação entre os três corpos de prova coletados, mostrando uma uniformidade da solda ao longo da área soldada. Este resultado também foi obtido pela análise microestrutural. Como a diferença entre as amostras S6 e S7 foi o tempo de união, 60 e 150 min, respectivamente, então é preferível empregar os parâmetros da amostra S6, visto que se obtém uma solda de qualidade em menos tempo. Por outro lado, mesmo que a amostra S8 leve mais tempo que S6, 150 min, a pressão empregada é menor, e para algumas aplicações, como aquelas em que se utilizam fornos menores, isso pode ser um fator determinante.

Desta forma pode-se afirmar que os conjuntos de parâmetros utilizados para a fabricação das amostras S6 e S8 podem ser considerados os mais adequados para a união por difusão do aço inoxidável 316L e que, portanto devem ser utilizados na fabricação de equipamentos como os trocadores de calor compactos. Como a resistência mecânica das amostras foi da mesma ordem de grandeza da resistência do metal base, estima-se que o equipamento apresente uma grande confiabilidade para operar em altas pressões, podendo utilizar a própria resistência mecânica do metal base nos cálculos da pressão máxima de trabalho.

\section{Conclusão}

No presente trabalho foi realizado um estudo experimental para avaliar a influência dos três parâmetros: temperatura, pressão e tempo, na resistência mecânica da união por difusão do aço inoxidável 316L.

Na primeira parte do estudo, foi realizada uma análise da influência dos parâmetros na resistência mecânica das amostras unidas. Com base nos resultados de tração, verificou-se que o parâmetro temperatura foi o que apresentou a maior influência na qualidade da união.

Na segunda parte do estudo focou-se na análise microestrutural das amostras produzidas, onde se pôde constatar que a interface de união estava mais evidente nas amostras S1 a S5 e S9, evidenciando a baixa qualidade da solda. Por outro lado, a interface de união das amostras S6, S7 e S8 é quase imperceptível, mostrando uma maior continuidade de material na região ligada, em comparação com as outras.

Na terceira parte do estudo, identificou-se o conjunto de parâmetros que apresentou os melhores resultados, visando futuras aplicações. Constatou-se que as amostras S6, S7 e S8 apresentaram maior resistência mecânica a tração, da mesma ordem de grandeza dos corpos de prova do metal base, apresentando ainda, pequena variação entre os três corpos de prova, mostrando uma uniformidade da união ao longo da interface unida, como também evidenciado na análise microestrutural.

Desse modo, comparando essas três amostras, pode-se concluir que os parâmetros utilizados nas amostras $\mathrm{S} 6\left(1040{ }^{\circ} \mathrm{C}\right.$, 9,51 MPa e $60 \mathrm{~min})$ e $\mathrm{S} 8\left(104{ }^{\circ} \mathrm{C}, 7,98 \mathrm{MPa}\right.$ e $\left.150 \mathrm{~min}\right)$, que apresentam menores tempo e pressão, respectivamente, podem ser considerados os mais adequados para a união de aços inoxidáveis AISI 316L e portanto podem ser empregados na fabricação de trocadores de calor compactos unidos por difusão.

Os resultados comprovaram que a escolha apropriada dos parâmetros de união está diretamente relacionada com a qualidade da união, podendo a interface de ligação apresentar propriedades similares às do próprio metal base.

\section{Referências}

[1] An ZL, Luan W-L, Xuan FZ, Tu ST. High temperature performance of 316L-SS joint produced by diffusion bonding. Key Engineering Materials. 2005;297-300:2795-2799. http://dx.doi.org/10.4028/www.scientific.net/KEM.297-300.2795.

[2] Brandner JJ, Bohn L, Henning T, Schygulla U, Schubert K. Microstructure heat exchanger applications in laboratory and industry. Heat Transfer Engineering. 2007;28(8-9):761-771. http://dx.doi.org/10.1080/01457630701328528.

[3] Li Y, Xuan F, Li S, Tu S. Quality evaluation of diffusion bonded joints by electrical resistance measuring and microscopic fatigue testing. Chinese Journal of Mechanical Engineering. 2011;24(02):1. http://dx.doi.org/10.3901/CJME.2011.02.187.

[4] Hesselgreaves JE. Compact heat exchangers: selection, design, and operation. Amsterdam: Pergamon; 2001. 
[5] Bouquet N, Rigal E, Grenoble SC, Bernard F, Dijon OH. Interface formation during HIP-bonding of austenitic stainless steel. In: Proceedings of the Brazing, High Temperature Brazing and Diffusion Bonding: Lectures and Posters of the 10th International Conference - LÖT; 2013 June 18-20; Aachen. Düsseldorf: DVS Media; 2013. p. 118-122.

[6] Mylavarapu SK, Sun X, Glosup RE, Christensen RN, Patterson MW. Thermal hydraulic performance testing of printed circuit heat exchangers in a high-temperature helium test facility. Applied Thermal Engineering. 2014;65(1-2):605-614. http://dx.doi.org/10.1016/j.applthermaleng.2014.01.025.

[7] Mylavarapu SK, Sun X, Christensen RN, Unocic RR, Glosup RE, Patterson MW. Fabrication and design aspects of high-temperature compact diffusion bonded heat exchangers. Nuclear Engineering and Design. 2012;249:49-56. http://dx.doi.org/10.1016/j.nucengdes.2011.08.043.

[8] Li Q, Flamant G, Yuan X, Neveu P, Luo L. Compact heat exchangers: A review and future applications for a new generation of high temperature solar receivers. Renewable \& Sustainable Energy Reviews. 2011;15(9):4855-4875. http://dx.doi.org/10.1016/j.rser.2011.07.066.

[9] Mortean MVV, Cisterna LHR, Paiva KV, Mantelli MBH. Development of diffusion welded compact heat exchanger technology. Applied Thermal Engineering. 2016;93:995-1005. http://dx.doi.org/10.1016/j.applthermaleng.2015.09.021.

[10] Hill A, Wallach ER. Modelling solid-state diffusion bonding. Acta Metallurgica. 1989;37(9):2425-2437. http://dx.doi.org/10.1016/00016160(89)90040-0.

[11] An ZL, Tu ST. Mechanical performances and failure modes of direct diffusion bonding joints of 316L stainless steel. Key Engineering Materials. 2006;324-325:979-982. http://dx.doi.org/10.4028/www.scientific.net/KEM.324-325.979.

[12] Li S-X, Xuan F-Z, Tu S-T. Fatigue damage of stainless steel diffusion-bonded joints. Materials Science and Engineering A. 2008;480(12):125-129. http://dx.doi.org/10.1016/j.msea.2007.06.062.

[13] Li S-X, Li L, Yu S-R, Akid R, Xia H-B. Investigation of intergranular corrosion of 316L stainless steel diffusion bonded joint by electrochemical potentiokinetic reactivation. Corrosion Science. 2011;53(1):99-104. http://dx.doi.org/10.1016/j.corsci.2010.09.027.

[14] Mortean MVV, Buschinelli AJA, Paiva KV, Mantelli MBH, Remmel J. Soldagem por difusão de aços inoxidáveis para fabricação de trocadores de calor compactos. Soldagem e Inspeção. 2016;21(1):103-114. http://dx.doi.org/10.1590/0104-9224/SI2101.10.

[15] Pavlova N. Aplicação do processo de soldagem por difusão na união de componentes inoxidáveis para uso espacial [dissertação de mestrado]. São José dos Campos: Instituto Tecnológico de Aeronáutica; 2005.

[16] American Society for Testing and Materials. ASTM E8/E8M - 15a: standards test methods for tension testing of metallic materials. West Conshohocken: ASTM; 2015.

[17] Callister WD Jr, Soares SMS. Ciência e engenharia de materiais: uma introdução. Rio de Janeiro: Livros Técnicos e Científicos; 2008.

[18] American Society for Testing and Materials. ASTM A240 / A240M - 17: standard specification for chromium and chromium-nickel stainless steel plate, sheet, and strip for pressure vessels and for general applications. ASTM; 2017.

http://dx.doi.org/10.1520/A0240_A0240M-17. 